\title{
Revisão de Estudos Sobre o Picture Exchange Communication System (PECS) Para o Ensino de Linguagem a Indivíduos com Autismo e Outras Dificuldades de Fala
}

ReVIeW of Studies on the Picture Exchange Communication System

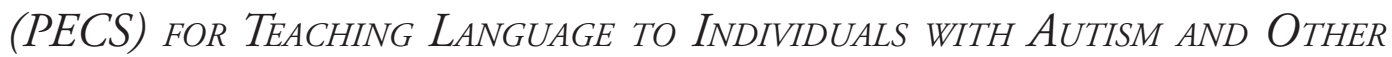
SPEECH DIFFICULTIES

Táhcita Medrado MIZAEL ${ }^{1}$ Ana Lúcia Rossito AIELLO ${ }^{2}$

RESUMO: o Picture Exchange Communication System (PECS) é um sistema de comunicação frequentemente utilizado em indivíduos com autismo e/ou pouca fala funcional. Até a data, nenhuma revisão sobre intervenções com o PECS foi publicada no Brasil. Assim, os objetivos deste estudo foram revisar as literaturas brasileira e estrangeira sobre o estado da arte do PECS como um instrumento de ensino de linguagem a indivíduos com autismo e dificuldades de fala, apontar o que tem sido pesquisado, os resultados mais encontrados e direçôes para novos estudos, principalmente para a literatura brasileira. Quatro artigos foram encontrados na literatura estrangeira e seis na literatura brasileira. Dados referentes ao número de participantes, delineamento de pesquisa, duração do estudo, setting, fases implementadas, resultados encontrados, fidedignidade inter-observador e de procedimento, validade social, manutenção e generalizaçáo foram sumarizados. Os estudos revisados mostraram que, em consonância com a literatura, o PECS parece ser efetivo no ensino da comunicação a indivíduos com autismo ou com pouca fala funcional, apesar de o pequeno número de participantes de cada estudo indicar que o PECS é uma estratégia de ensino individual.

PALAVRAS-CHAVE: Educação Especial. PECS. Autismo. Distúrbios da comunicação.

\begin{abstract}
The Picture Exchange Communication System (PECS) is a communication system frequently used with people with autism and/or with little functional speech. To date, no review has been published on PECS interventions in the Brazilian literature. Thus, the aims of this study were to review Brazilian and foreign literature on the state of art of PECS as a language teaching instrument for individuals with autism and speech difficulties, and to point out what has been researched, the main results and directions for further research, especially regarding Brazilian literature. Four articles were found in foreign literature and six in the Brazilian one. Data on the number of participants, research design, study duration, setting, implemented phases, main outcomes, inter-observer reliability and procedure data, social validity, generalization and maintenance data were summarized. In consonance with the existing literature, the reviewed studies show that PECS seems to be effective in teaching communication to individuals with autism or little functional speech, despite the small number of participants in each study, suggesting that PECS is an individual teaching strategy.
\end{abstract}

KEYWORDS: Special Education. Picture Exchange Communication System. Autism. Communication disorders.

\section{INTRODUÇÁO}

O autismo é um transtorno global do desenvolvimento (TGD), também conhecido como transtorno do espectro autista (TEA), principalmente na literatura internacional, caracterizado por dificuldades na interação social e na comunicação verbal e não-verbal e por comportamentos e interesses bastante restritos e repetitivos, o que é conhecido como a tríade de prejuízos (ASSOCIAÇÃO AMERICANA DE PSICOLOGIA, 2003).

\footnotetext{
${ }^{1}$ Mestranda em Psicologia/Universidade Federal de São Carlos (UFSCar), São Carlos, São Paulo, Brasil. tahcitammizael@ibest. com.br

${ }^{2}$ Doutora em Psicologia, Docente da Universidade Federal de São Carlos (UFSCar), Departamento de Psicologia. São Carlos, São Paulo, Brasil.ana.aiello@terra.com.br
} 
Walter (2007) relata que 70 a 80\% dos indivíduos autistas não demonstram qualquer tipo de comunicação verbal ou fala com funçóes comunicativas. Sem o uso de comunicação de forma funcional, indivíduos com ou sem autismo se frustram e sentem que falharam em seus objetivos de se comunicar (COWAN; ALLEN, 2007). Assim, uma vez que a comunicação é a peça chave para relacionar-se com os outros, métodos alternativos de ensino da linguagem acabam se tornando de suma importância para essas pessoas (NUNES, 2009). Os métodos de comunicação alternativa e ampliada (CAA) podem ser definidos como um conjunto de métodos e técnicas que possibilitam a comunicação a indivíduos sem ou com pouca fala funcional (NUNES, 2003; 2008). Atualmente, existe uma diversidade de métodos de CAA. O Picture Exchange Communication System é um desses sistemas, bastante utilizado em indivíduos com autismo e/ou outras dificuldades de fala.

\subsection{PECS: UMA ALTERNATIVA DE INTERVENÇÃO NA ÁREA DE LINGUAGEM COM PESSOAS COM AUTISMO}

O Picture Exchange Communication System (PECS) é um sistema de comunicação que ressalta a relação interpessoal, em que ocorre um ato comunicativo entre o indivíduo com dificuldades de fala e um adulto, por meio de trocas de figuras. O treino com o PECS se dá viaseis fases, que são: 1) Fazer pedidos através da troca de figuras pelos itens desejados; 2) Ir até a tábua de comunicação, apanhar uma figura, ir a um adulto e entregá-la em sua mão; 3) Discriminar entre as figuras; 4) Solicitar itens utilizando várias palavras em frases simples, fixadas na tábua de comunicação; 5) Responder à pergunta O que você quer; 6) Emitir comentários espontâneos (BONDY; FROST, 2001).

Atualmente, existem diversos programas de CAA para indivíduos com autismo e/ou dificuldades de fala, embora não haja, até o momento, um guia para clínicos que determine qual estratégia e/ou sistema de comunicação seja o mais efetivo para indivíduos com autismo e/ou outras dificuldades de fala (FLIPPIN; RESKA; WATSON, 2010). Como ainda não se sabe qual sistema de CAA é o mais efetivo, pesquisas, como as revisóes bibliográficas, mostram sua importância na organização dos dados existentes sobre a implementação e a intervenção dos diferentes sistemas de comunicação. Por meio das revisóes, podemos avaliar e comparar diferentes métodos de comunicação, apontar lacunas e direçóes para a pesquisa futura, e ainda comparar o estado da arte em diferentes países, como, por exemplo, o Brasil e os Estados Unidos.

$\mathrm{Na}$ literatura estrangeira, encontramos duas revisóes publicadas sobre intervençóes com o PECS (entre julho de 2009 e agosto de 2011). A pesquisa de Preston e Carter (2009) revisou27 estudos e revelou que, embora vários artigos apresentassem dados insuficientes para uma avaliação completa dos ganhos com o PECS, os estudos revisados são prova preliminar de que o PECS pode ser eficaz para crianças e adultos com TEA e outros problemas na fala, e que os benefícios na área da comunicação são evidentes. Todavia, a falta de pesquisas que tenham um controle experimental claro e que utilizem tentativas de controle randomizado (RCTs) ainda faz com que os resultados obtidos nos estudos analisados não tenham validade ou precisão adequadas para se afirmar que os resultados foram devidos ao uso do PECS e não de outras variáveis intervenientes. 
A segunda revisão, uma meta-análise publicada em 2010 por Flippin et al., encontrou 11 artigos, e mostrou aumentos na comunicação dos participantes. Entretanto, os resultados quanto à fala espontânea foram mistos (entre os estudos de sujeito único, foram apontados ganhos e entre os estudos com delineamento de grupo, os resultados foram questionáveis). Contudo, de maneira geral, os estudos demonstraram que o PECS é promissor como prática baseada em evidência, porém, ainda não estabelecida, na promoção da comunicação de crianças com autismo.

Vemos então, que, de acordo com essas revisóes, na literatura estrangeira, vários estudos foram publicados no que se refere ao ensino do PECS com o objetivo de comunicação, entre outros. Como está, entretanto, o estado da arte da pesquisa brasileira? Houve na pesquisa estrangeira novos estudos a partir da última revisão publicada em 2010? Os objetivos deste estudo foram revisar a bibliografia estrangeira (artigos em inglês)e a brasileira sobre o PECS como instrumento de ensino de linguagem a indivíduos com autismo e outras dificuldades de fala, apontar o que tem sido pesquisado, os principais resultados e mostrar direçóes para novos estudos, principalmente no que se refere à literatura brasileira.

\section{Desenvolvimento}

\subsection{Literatura Estrangeira}

Artigos foram buscados nas seguintes bases: Periódicos CAPES e BIREME, utilizando os descritores PECS e Picture Exchange Communication System no título. Os estudos foram incluídos se fossem pesquisas em inglês publicadas entre 1 de julho de 2009 (período posterior à última revisão encontrada até a data do presente estudo) e 31 de agosto de 2011 (período da coleta de dados da presente pesquisa); descrevessem uma intervenção com o uso do PECS e utilizassem o PECS proposto por Bondy e Frost (e não adaptações deste) como parte ou toda a intervenção.Artigos que utilizaram modelos baseados no PECS foram excluídos.

Foram encontrados 14 artigos, entretanto, 10 foram excluídos por não se enquadrarem nos critérios de inclusão. Portanto, 4 artigos foram revisados.

\subsection{Literatura Brasileira}

Os artigos foram buscados nas seguintes bases de dados: SciELO, PePSIC, BIREME e Banco de Teses e Dissertações CAPES, com as palavras-chave: PECS, Picture Exchange Communication System, Autismo e PECS em qualquer campo. Não foi delimitado um período de tempo, uma vez que, até a data, não há nenhuma publicação sobre intervenções com o PECS. Todos os artigos encontrados que relataram parte ou toda a intervenção com o PECS foram incluídos. Artigos que utilizaram adaptaçóes ou modificações do PECS também foram incluídos.

Segundo esses critérios, foram localizados 6 artigos.

\subsection{ANÁLISE}

Foi realizada uma revisão sistematizada (systematized review, GRANT; BOOTHT, 2009), que é caracterizada pela inclusão de alguns elementos presentes na revisão sistemática, entretanto sem afirmar que o resultado da busca é uma revisão sistemática. Geralmente, nesse 
tipo de revisão, os artigos encontrados não são simplesmente catalogados: eles são organizados de maneira sistemática.

Foram sumarizados, para cada artigo encontrado de ambas as literaturas, dados referentes ao objetivo do estudo, número de participantes, delineamento de pesquisa, duração do estudo, setting, fases implementadas, resultados encontrados, dados de fidedignidade interobservador e de procedimento, validade social ${ }^{3}$ e dados de manutenção ${ }^{4}$ e generalização ${ }^{5}$. A escolha dos dados para análise e para exibição nos quadros foi feita com base nos dados analisados pelas duas revisóes encontradas na literatura estrangeira (PRESTON; CARTER, 2009; FLIPPIN et al., 2010) sobre o PECS.

\section{LITERATURA ESTRANGEIRA}

Uma síntese com os autores, o número de participantes, o diagnóstico, a idade dos participantes, o delineamento de pesquisa, os dados de manutenção e generalização, as fases ensinadas e as variáveis dependentes estão no Quadro 1. Em seguida, estão sumariados dados referentes aos participantes, delineamento de pesquisa, dados de manutenção e generalizaçáo, duração dos estudos e fases ensinadas, setting, dados de fidedignidade de procedimento e de acordo inter-observador, validade social e resultados encontrados.

\subsection{Participantes}

Dois dos quatro estudos (YODER; LIEBERMAN, 2010; MALHOTRA; RAJENDER; BHATIA; SINGH, 2010) utilizaram medidas padronizadas para avaliar as habilidades dos participantes antes dos estudos.

\subsection{Delineamento de Pesquisa}

Dos quatro artigos analisados, três são considerados pesquisas experimentais. A pesquisa de Malhotra et al.(2010) não explicitou o tipo de delineamento utilizado.

\subsection{Dados de Manutenção e de Generalização}

Apenas dois estudos relataram dados de manutenção. Na pesquisa de Ganz, Parker e Benson (2009), os dados foram obtidos após cinco, seis, 11 e 23 semanas depois do final da intervenção. Um dos participantes utilizou nove figuras após seis semanas da intervenção e oito figuras 23 semanas após a intervenção, mostrando que os ganhos foram mantidos por um longo período após o treino. Um segundo participante utilizou oito figuras após cinco semanas do fim do treino e nove figuras após 11 semanas da última intervenção, mostrando um aumento no número de figuras utilizadas. Um terceiro participante utilizou cinco figuras

\footnotetext{
${ }^{3}$ Validade social se refere à importância social dos objetivos, procedimentos e métodos propostos por intervençóes e de seus resultados (FOSTER; MASH, 1999), ou seja, à aplicabilidade do que foi aprendido durante as intervençốes na vida social dos indivíduos.

${ }^{4}$ Manutenção se refere à extensão na qual o aprendiz continua a emitir os comportamentos aprendidos após o fim de uma intervenção que ensinou esses comportamentos (CATANIA, 1999).

${ }^{5}$ Generalização diz respeito à emissão de uma mesma resposta ou classe de respostas diante de estímulos diferentes, ou seja, é a capacidade de emitir as habilidades aprendidas em ambientes que não eram utilizados na intervenção, e/ou com indivíduos que não ensinaram essas habilidades (CATANIA, 1999).
} 
seis semanas após o último treino, utilizando fala funcional em metade do tempo da sessão de manutenção. Na pesquisa de Malhotra et al., (2010), os ganhos do participante foram mantidos 3 meses após o término do treino.

Dois estudos reportaram dados de generalização. No estudo de Ganz et al.(2009), os dados mostraram que os três participantes generalizaram o uso das figuras para um adulto que não havia trabalhado extensivamente com nenhum dos participantes, e no estudo de Chaabane, Alber-Morgan e Debar (2009) os dois participantes generalizaram o uso de figuras para itens não treinados.

Quadro 1 - Sumário dos estudos encontrados na literatura estrangeira.

\begin{tabular}{|c|c|c|c|c|c|c|c|}
\hline Autores & $\begin{array}{l}\text { Número de } \\
\text { Participantes }\end{array}$ & Diagnóstico & $\begin{array}{c}\text { Idade dos Partici- } \\
\text { pantes }\end{array}$ & $\begin{array}{l}\text { Delineamento da } \\
\text { Pesquisa }\end{array}$ & $\begin{array}{l}\text { Dados de } \\
\text { Manuten- } \\
\text { ção }(M) \text { ou } \\
\text { Generaliza- } \\
\text { çăo }(G) \\
\end{array}$ & $\begin{array}{c}\text { Fases Ensi- } \\
\text { nadas }\end{array}$ & $\begin{array}{l}\text { Variável Depen- } \\
\text { dente }\end{array}$ \\
\hline $\begin{array}{l}\text { Yoder e Lieberman } \\
(2010)\end{array}$ & 19 & $\begin{array}{l}\text { Autismo ou } \\
\text { Transtorno } \\
\text { do Espectro } \\
\text { Autista }\end{array}$ & $1 \mathrm{a} 6 \mathrm{~m}-5 \mathrm{a}$ & $\begin{array}{l}\text { Tentativas de } \\
\text { Controle Rando- } \\
\text { mizado (RCT) }\end{array}$ & |---- & ----- & $\begin{array}{l}\text { Uso do PECS sem } \\
\text { o uso concomitante } \\
\text { de gestos, vocaliza- } \\
\text { çóes e palavras }\end{array}$ \\
\hline $\begin{array}{l}\text { Ganz, Parker e } \\
\text { Benson (2009) }\end{array}$ & 3 & Autismo & $3-6 a$ & $\begin{array}{l}\text { Linha de Base } \\
\text { com Múltiplas } \\
\text { Provas }\end{array}$ & $\mathrm{M} / \mathrm{G}$ & I & $\begin{array}{l}\text { Uso das figuras, } \\
\text { uso de palavras e } \\
\text { comportamento } \\
\text { mal-adaptativo }\end{array}$ \\
\hline $\begin{array}{l}\text { Malhotra, } \\
\text { Rajender, Bhatia e } \\
\text { Singh(2010) }\end{array}$ & 1 & Autismo & $7 \mathrm{a}$ & ---- & M & I-VI & $\begin{array}{l}\text { Melhora na comu- } \\
\text { nicação, habilidades } \\
\text { de autoajuda e } \\
\text { diminuiçáo de } \\
\text { problemas de } \\
\text { comportamento }\end{array}$ \\
\hline $\begin{array}{l}\text { Chaabane, Alber- } \\
\text { Morgan e DeBar } \\
(2009)\end{array}$ & 2 & TEA & $5-6 a$ & \begin{tabular}{|l|} 
Linha de Base \\
Múltipla entre \\
categoria de sím- \\
bolos (cor, forma \\
e funçóes)
\end{tabular} & G & ---- & $\begin{array}{l}\text { Respostas corretas, } \\
\text { respostas incorretas } \\
\text { e quando não havia } \\
\text { resposta }\end{array}$ \\
\hline
\end{tabular}

Fonte: Base de dados da pesquisa.

\subsection{Duração dos Estudos e Fases Ensinadas}

Apenas dois artigos relataram a duração do estudo (YODER; LIEBERMAN, 2010; MALHOTRA et al., 2010). No primeiro estudo, a duração foi de seis meses, embora o estudo não explicitasse qual o número de fases ensinado aos participantes. O segundo estudo teve a duração de três meses e ensinou todas as seis fases do PECS.

\subsection{SETTING}

Três dos quatro estudos relataram o ambiente no qual a pesquisa foi realizada. O estudo de Chaabane et al. (2009) foi feito na casa dos participantes; o de Yoder e Lieberman (2010) na clínica de uma Universidade e o de Ganz et al. (2009) em um escritório e sala de aula da escola na qual os participantes estudavam. 


\subsection{Dados de Fidedignidade de Procedimento e de Acordo Inter-Observador}

Dados sobre o acordo inter-observador foram reportados em três dos quatro estudos (CHAABANE et al., 2009; GANZ et al., 2009; YODER; LIEBERMAN, 2010). Nestes estudos, os dados foram coletados em, no mínimo, $20 \%$ das sessóes de intervenção, chegando a $80 \%$ em alguns casos (por exemplo, GANZ et al., 2009), e o acordo inter-observador variou de 67 a $100 \%$.

Três pesquisas relataram dados de fidedignidade de procedimento. No estudo de Ganz et al. (2009), a média de fidelidade de tratamento durante a intervenção foi de 99,33\%; na pesquisa de Yoder e Liberman (2010) foi de 2,88 (um era considerado pobre, dois era considerado bom e três era considerado excelente) e no estudo de Chaabane et al. (2009), a média foi de $97,5 \%$.

\subsection{Validade Social}

Apenas um estudo relatou dados de validade social (CHAABANE et al., 2009), que foram aferidos por meio de um questionário, no qual os pais e outras pessoas significativas dos participantes foram orientados a registrar qualquer improvisaçáo de mandos (nesse caso, pedidos) para itens ou ambientes não-treinados.

\subsection{Resultados Encontrados}

De modo geral, os resultados indicam que o PECS foi eficaz em aumentar o número de figuras utilizadas de maneira independente (GANZ et al., 2009; YODER; LIEBERMAN, 2010), aumentar a fala inteligível (GANZ et al., 2009) e diminuir os movimentos repetitivos com a cabeça e o comportamento de agitar as mãos - comportamentos-problema (MALHOTRA et al., 2010).

\section{Literatura BRASILEIRA}

Os autores, o número de participantes, o diagnóstico, a idade dos participantes, o delineamento de pesquisa, os dados de manutenção e generalização, as fases ensinadas e as variáveis dependentes estão sumarizados no Quadro 2. Em seguida, estáo sumariados dados referentes aos participantes, delineamento de pesquisa, dados de manutenção e generalização, duração dos estudos e fases ensinadas, setting, dados de fidedignidade de procedimento e de acordo inter-observador, validade social e resultados encontrados.

\subsection{Participantes}

Apenas dois dos seis estudos encontrados (ALMEIDA; PIZA; LAMÔNICA, 2005; FERREIRA; TEIXEIRA; BRITO, 2011) utilizaram medidas padronizadas para avaliar as habilidades dos participantes antes das intervençôes realizadas. 


\subsection{Delineamento de Pesquisa}

Em três estudos (WALTER, 2000; ALMEIDA et al., 2005; FIDALGO; GODOI; GIOIA, 2008), o delineamento utilizado foi $A B$, sendo que no estudo de Walter (2000), houve replicação do delineamento em quatro participantes. Uma pesquisa (FERREIRA et al., 2011) consistiu em um estudo de caso do tipo longitudinal. Apenas duas pesquisas utilizaram delineamento experimental, no caso, linha de base múltipla (PIZA, 2002; LEITE, 2005).

\subsection{Dados de Manutençáo e de Generalizaçáo}

Nenhum estudo relatou dados de manutenção dos ganhos. Todavia, dois dos seis estudos relataram dados de generalizaçáo. No estudo de Leite (2005), três funcionárias da escola realizaram os testes de generalização a pedido da pesquisadora, a qual não conduziu os testes e não esteve presente na escola durante sua realizaçáo para evitar servir de elo entre os ambientes e, portanto, promover a generalizaçáo. O participante generalizou em 90 a 100\% dos itens solicitados nas 10 sessóes de testes de generalização realizados na escola e na residência da criança. No estudo de Ferreira et al. (2011), o participante demonstrou ter iniciado o processo de generalização da comunicação, uma vez que, em alguns momentos, solicitou, em ambientes variados e de forma espontânea através da comunicação alternativa, objetos de seu interesse.

Quadro 2 - Sumário dos estudos encontrados na literatura brasileira.

\begin{tabular}{|c|c|c|c|c|c|c|c|}
\hline Autores & $\begin{array}{l}\text { Número } \\
\text { de Parti- } \\
\text { cipantes }\end{array}$ & $\begin{array}{l}\text { Diagnós- } \\
\text { tico }\end{array}$ & $\begin{array}{l}\text { Idade dos } \\
\text { Partici- } \\
\text { pantes }\end{array}$ & $\begin{array}{l}\text { Delineamento da } \\
\text { Pesquisa }\end{array}$ & $\begin{array}{c}\text { Dados de Ma- } \\
\text { nutençáo }(\mathrm{M}) \\
\text { ou Generaliza- } \\
\text { çáo }(\mathrm{G})\end{array}$ & $\begin{array}{l}\text { Fases Ensi- } \\
\text { nadas }\end{array}$ & Variável Dependente \\
\hline Walter (2000) & 4 & Autismo & $5-8 a$ & $\begin{array}{l}\text { AB replicado em } \\
\text { quatro partici- } \\
\text { pantes }\end{array}$ & ---- & $\begin{array}{l}5 \text { fases do } \\
\text { PECS- } \\
\text { Adaptado }\end{array}$ & $\begin{array}{l}\text { Desempenho dos } \\
\text { participantes em } \\
\text { cada sessão (+ tipo de } \\
\text { ajuda) e vocabulário } \\
\text { emitido nas sessóes }\end{array}$ \\
\hline Piza (2002) & 3 & $\begin{array}{l}\text { Paralisia } \\
\text { Cerebral } \\
\text { Severa }\end{array}$ & 6-11a & $\begin{array}{l}\text { Linha de Base } \\
\text { Múltipla }\end{array}$ & ----- & $\begin{array}{l}5 \text { fases do } \\
\text { PECS- } \\
\text { Adaptado }\end{array}$ & $\begin{array}{l}\text { Desempenho dos } \\
\text { participantes em } \\
\text { cada sessão (+ tipo de } \\
\text { ajuda) e vocabulário } \\
\text { emitido nas sessóes }\end{array}$ \\
\hline $\begin{array}{l}\text { Almeida, Piza } \\
\text { e Lamônica } \\
(2005)\end{array}$ & 1 & $\begin{array}{l}\text { Paralisia } \\
\text { Cerebral }\end{array}$ & $9 \mathrm{a} 10 \mathrm{~m}$ & $\mathrm{AB}$ & ---- & $\begin{array}{l}5 \text { fases do } \\
\text { PECS- } \\
\text { Adaptado }\end{array}$ & $\begin{array}{l}\text { Número de trocas com } \\
\text { ou sem êxito }\end{array}$ \\
\hline Leite (2005) & 1 & Autismo & $7 \mathrm{a}$ & $\begin{array}{l}\text { Linha de } \\
\text { Base Múltipla } \\
\text { entre Ambientes }\end{array}$ & G & I-III & $\begin{array}{l}\text { Frequência de ocorrên- } \\
\text { cia de mandos e de vo- } \\
\text { calizaçóes inteligíveis }\end{array}$ \\
\hline $\begin{array}{l}\text { Fidalgo, } \\
\text { Godoi e Gioia } \\
(2008)\end{array}$ & 2 & $\begin{array}{l}\text { P1: Sín- } \\
\text { drome do } \\
\text { X-Frágil } \\
\text { P2: Sín- } \\
\text { drome de } \\
\text { Down } \\
\end{array}$ & $\begin{array}{l}\text { P1: 11a } \\
\text { P2: } 23 a\end{array}$ & $\mathrm{AB}$ & ---- & I & $\begin{array}{l}\text { Frequência e função } \\
\text { das verbalizaçōes + } \\
\text { frequência de outras } \\
\text { respostas específica. }\end{array}$ \\
\hline $\begin{array}{l}\text { Ferreira, Tei- } \\
\text { xeira e Brito } \\
(2011)\end{array}$ & 1 & Autismo & $20 \mathrm{a}$ & $\begin{array}{l}\text { Estudo de caso do } \\
\text { tipo longitudinal }\end{array}$ & G & $\begin{array}{l}\text {---- (PECS- } \\
\text { Adaptado) }\end{array}$ & $\begin{array}{l}\text { Número de atos } \\
\text { comunicativos expres- } \\
\text { sos por minuto nas } \\
\text { avaliaçóes }\end{array}$ \\
\hline
\end{tabular}

Fonte: Base de dados da pesquisa. 


\subsection{Duraçáo dos Estudos e Fases Ensinadas}

Quatro pesquisas reportaram a duração do estudo. As pesquisas de Piza (2002), Almeida et al. (2005) e a de Ferreira et al. (2011) duraram nove meses cada. A pesquisa de Walter (2000) durou aproximadamente dois anos. As quatro pesquisas utilizaram o PECSAdaptado (adaptação do PECS, baseada no Curriculum Funcional Natural ${ }^{6}$ ) e ensinaram as cinco fases do PECS-Adaptado, exceto a pesquisa de Ferreira et al. (2011), que não explicitou o número de fases ensinado.

\subsection{SETTING}

Todos os seis estudos relataram a sala de aula como um dos ambientes nos quais suas pesquisas ocorreram. Além deste ambiente, foram relatados a casa do participante (LEITE, 2005), ambientes da comunidade onde o participante estudava, como a padaria e o supermercado (WALTER, 2000), uma clínica particular (FIDALGO et al., 2008), entre outros ambientes.

\subsection{Dados de Fidedignidade de Procedimento e de Acordo Inter-Observador}

Os dados de acordo inter-observador foram relatados em três das seis pesquisas. $\mathrm{Na}$ pesquisa de Walter (2000), o índice de fidedignidade obteve uma média de 95,75\%. Piza (2002) relatou um índice de fidedignidade médio de 93,33\%, e o estudo de Almeida et al. (2005) relatou um índice de fidedignidade de 95\%. Nestes estudos, os dados foram coletados em, no mínimo, 20\% das sessóes. O estudo de Leite (2005) não relatou o valor do índice de fidedignidade, embora relatasse o ter realizado.

Dois estudos (WALTER, 2000; PIZA, 2002) descreveram os dados de acordo interobservador entre todos os sujeitos e condiçóes (linha de base e intervenção, manutenção e generalizaçáo, se houvessem), e nenhum estudo relatou dados de fidedignidade de procedimento.

\subsection{Validade Social}

Nenhuma pesquisa relatou dados sobre a validade social de seus estudos.

\subsection{Resultados Encontrados}

De maneira geral, os resultados das seis pesquisas indicam que o PECS e o PECSAdaptado foram eficazes no ensino da comunicação funcional pelos participantes, com o uso tanto gestual quanto vocal destes, aumentando o número de trocas de figuras de maneira independente (WALTER, 2000; PIZA, 2002; ALMEIDA et al., 2005; LEITE, 2005; FIDALGO et al., 2008; FERREIRA et al., 2011), aumentando o número de vocalizaçóes

${ }^{6}$ O PECS-Adaptado consiste em uma adaptação do uso do PECS, aplicado ao contexto do Curriculum Funcional Natural. Suas principais diferenças entre ele e o PECS original são o número de fases (6 no PECS original e 5 no PECS-Adaptado), um número maior de falas do treinador para o aluno e a introdução de algumas palavras da cultura brasileira, como feijoada e pamonha (WALTER, 2000). 
com intenção comunicativa ou a fala funcional (WALTER, 2000; PIZA, 2002; LEITE, 2005; FERREIRA et al., 2011) e até diminuindo comportamentos inadequados (WALTER, 2000).

Retomando, os objetivos deste trabalho foram revisar a bibliografia estrangeira e a brasileira a respeito de intervençóes com o PECS como instrumento de ensino de linguagem a indivíduos com autismo e/ou dificuldades de fala, indicar o que tem sido pesquisado e os resultados destas pesquisas. Além disso, também se buscou indicar direçôes para estudos futuros, principalmente no que se refere à literatura brasileira.

Inicialmente, é importante relatar que os quatro artigos encontrados na literatura estrangeira são apenas um recorte de uma gama de estudos já realizados, que apontam para a eficácia do PECS como um método de ensino de linguagem para pessoas com TGD/TEA e dificuldades de fala em geral (GANZ; SIMPSON, 2004; CARR; FELCE, 2007, etc.). Entretanto, como essa revisão buscou verificar o que havia de novo, após a publicação da última revisão sobre o PECS, o foco estará nos artigos encontrados.

Assim, os resultados das pesquisas revisadas mostram claramente que o PECS, em consonância com a literatura existente (WALTER, 2000; GANZ; SIMPSON, 2004; CARR; FELCE, 2007; WALTER, 2007; GANZ; SIMPSON; CORBIN-NEWSOME, 2008, entre outros), parece se mostrar um método de ensino de linguagem eficaz, tanto na literatura estrangeira, quanto na brasileira. Em ambos os casos, pode-se verificar que o PECS foi eficaz na produção trocas de figuras feitas de maneira independente (WALTER, 2000; PIZA, 2002; ALMEIDA et al., 2005; LEITE, 2005; FIDALGO et al., 2008; GANZ et al., 2009; YODER; LIEBERMAN, 2010; FERREIRA et al., 2011), aumentando o número de vocalizaçóes com intenção comunicativa ou a fala funcional (WALTER, 2000; PIZA, 2002; LEITE, 2005; GANZ et al., 2009; FERREIRA et al., 2011) e diminuindo comportamentos-problema (WALTER, 2000; MALHOTRA et al., 2010).

$\mathrm{Na}$ maioria das pesquisas brasileiras (quatro), o método de ensino utilizado foi uma adaptação do PECS, baseada no Curriculum Funcional Natural, denominada PECSAdaptado (WALTER, 2000). Essa adaptação parece gerar os mesmos resultados positivos que o PECS original, ou seja, ambas as modalidades parecem ser efetivas no ensino de linguagem, e não só para indivíduos com autismo, uma vez que quatro crianças com Paralisia Cerebral (PIZA, 2002; ALMEIDA et al., 2005), um participante com Síndrome do X-Frágil e um adulto com Síndrome de Down (FIGALGO et al., 2008) também se beneficiaram do método. Entretanto, apesar dos ganhos obtidos, com crianças e adultos, portadores de TGD/TEA ou outras condiçôes, há uma série de fatores que devem ser averiguados mais de perto, objetivando melhorar a pesquisa acerca de métodos de ensino de linguagem.

$\mathrm{O}$ número de participantes na literatura estrangeira é maior do que na brasileira (vide Tabelas 1 e 2), entretanto, ainda é um número pequeno. Tal fato parece indicar que o PECS, apesar dos bons resultados obtidos nas pesquisas, não tem sido instrumento de ensino em escolas, não sendo uma ferramenta disponível para professores em sala de aula e não aplicado a grupos de indivíduos, o que favoreceria a aprendizagem de um maior número de indivíduos.

$\mathrm{Na}$ literatura estrangeira, dos quatro artigos revisados, apenas dois relataram a duração do estudo (YODER; LIEBERMAN, 2010; MALHOTRA et al., 2010). Em 
contrapartida, na literatura brasileira, quatro relataram o tempo de duração (WALTER, 2000; PIZA, 2002; ALMEIDA et al., 2005; FERREIRA et al., 2011). Não informar a duração do estudo compromete a própria pesquisa e outros pesquisadores que poderiam ter um melhor planejamento se soubessem a média de tempo utilizado para o treino, com base em estudos anteriores. Assim, a falta de dados e de definiçóes claras compromete a replicação das pesquisas e consequentemente sua qualidade.

Uma pesquisa estrangeira não relatou o setting utilizado (MALHOTRA et al., 2010). O desconhecimento do local onde ocorreram os treinos impossibilita uma visualização clara de como ocorreu o procedimento de pesquisa, além de impossibilitar a replicação dela.

Apenas quatro artigos (dois estrangeiros e dois brasileiros) utilizaram medidas padronizadas para avaliar as habilidades dos participantes antes das intervençóes (ALMEIDA et al., 2005; YODER; LIEBERMAN, 2010; MALHOTRA et al., 2010; FERREIRA et al., 2011). Como encontrar participantes semelhantes ao de determinado estudo se não há uma descrição clara das habilidades de cada um, medida por testes padronizados?

Com relação aos dados de fidedignidade de procedimento, a literatura estrangeira está à frente da brasileira: três dos quatro artigos relataram-na (CHAABANE et al., 2009; GANZ et al., 2009; YODER; LIEBERMAN, 2010), enquanto que, na literatura brasileira, nenhum trabalho a relatou. Se um procedimento não é confiável, ou seja, se não há avaliações de como o procedimento foi implementado efetivamente, não há garantias de que os dados obtidos sejam decorrentes daquele procedimento e não de outra variável.Apenas um artigo estrangeiro obteve dados sobre a validade social de sua pesquisa (CHAABANE et al., 2009), e na literatura brasileira, não há relatos sobre validade social. Isso diminui a aplicabilidade dos resultados, por não fornecer informaçóessobre se os resultados obtidos serão úteis na vida social dos participantes.

Os dados sobre manutenção e generalização também são de extrema importância. Saber se os ganhos com o treino serão mantidos a pequeno, médio e longo prazo e se eles serão utilizados com outras pessoas e/ou em outros ambientes, ou seja, seocorrerá generalização das habilidades aprendidas garante maior credibilidade ao método de ensino utilizado, consequentemente aumentando a possibilidade de que pais e professores queiram utilizar este método de ensino com seus filhos e alunos. Somente duas pesquisas estrangeiras relataram dados de manutenção das habilidades aprendidas (GANZ et al., 2009; MALHOTRA et al., 2010) enquanto que nenhuma pesquisa brasileira publicou dados de manutenção. Somente quatro estudos (dois brasileiros e dois estrangeiros) relataram dados de generalização (LEITE, 2005; CHAABANE et al., 2009; GANZ et al., 2009; FERREIRA et al., 2011).

Em uma perspectiva mais ampla, o delineamento de pesquisa revela muito sobre o índice de qualidade de uma pesquisa, informando dados aos leitores sobre suas limitaçóes e alcances, que variam de delineamento para delineamento. Assim, nesta revisão, foi encontrada, na literatura estrangeira, apenas uma pesquisa que utilizou tentativas de controle randomizado (YODER; LIEBERMAN, 2010), que é considerada por alguns autores como o "padrão ouro" das pesquisas que avaliam a intervenção clínica nas áreas de educação e medicina (PRESTON; CARTER, 2009). Além desta, mais duas pesquisas utilizaram um delineamento experimental, 
com o uso de linha de base múltipla (CHAABANE et al., 2009; GANZ et al., 2009). Entretanto, uma das pesquisas não relatou o tipo de delineamento utilizado (MALHOTRA et al., 2010).

$\mathrm{Na}$ literatura brasileira a situação é ainda mais precária. Apenas duas pesquisas utilizaram um delineamento experimental, no caso, linha de base múltipla (PIZA, 2002; LEITE, 2005), enquanto que três pesquisas empregaram um delineamento AB (WALTER, 2000; ALMEIDA et al., 2005; FIDALGO et al., 2008) e uma pesquisa realizou um estudo de caso (FERREIRA et al., 2011). O delineamento AB é o delineamento mais básico em pesquisas de sujeito único, no qual as mudanças ocorridas no comportamento-alvo são supostas como função da variável independente. Entretanto, como o efeito náo é replicado no mesmo participante, não há segurança para se dizer que a variável independente é a responsável pelas mudanças observadas. Todavia, vale ressaltar que as pesquisas dessa revisão envolvem estudos de intervenção, que, por sua vez, podem ser mais difíceis de implementar do que outros tipos de pesquisa, além de envolverem indivíduos com autismo, uma população reconhecidamente mais difícil de prover intervençóes. Com relação ao número de fases ensinadas vemos que, na literatura estrangeira, apenas duas das quatro pesquisas encontradas reportaram o número de fases ensinadas (GANZ et al., 2009; MALHOTRA et al., 2010); na literatura brasileira, cinco dos seis estudos reportaram o número de fases (WALTER, 2000; PIZA, 2002; ALMEIDA et al., 2005; LEITE, 2005; FIDALGO et al., 2008). Novamente, a falta de informação sobre o número de fases prejudica o entendimento do estudo como um todo, uma vez que cada uma das fases do PECS tem um objetivo, e algumas habilidades são treinadas somente em determinado momento. Assim, a ausência de dados sobre o que foi ensinado impede, entre outras coisas, a formulação de correlaçóes sobre a fase ensinada e os ganhos obtidos naquela fase. Por exemplo, a pesquisa de Flippin, et al. (2010) aponta que a fase quatro do PECS original pode ter uma papel na aquisição de fala espontânea, embora os poucos estudos existentes sobre o assunto limitem uma afirmação deste dado.

O número baixo de estudos e de participantes (em especial na literatura brasileira) dificulta uma análise mais completa dos dados, assim como as limitaçôes metodológicas já apontadas, que impedem afirmar que o PECS é realmente um promotor de linguagem gestual e vocal, embora haja prova preliminar indicando a eficácia do PECS. Sugere-se, portanto, para pesquisas futuras, tanto brasileiras quanto estrangeiras um maior rigor metodológico, com dados suficientes para a replicação dos estudos, melhor descrição do repertório comportamental de entrada dos participantes, maior uso de delineamentos com RCTs, testes de manutenção e de generalização dos ganhos obtidos e dados sobre a validade social das intervenções. Para a pesquisa brasileira, em especial, recomendamos um maior número de participantes, maior uso de delineamentos experimentais, para aumentar a validade interna e a execução de estudos comparativos, uma vez que nenhuma pesquisa comparou dois procedimentos de ensino. Sugere-se ainda, pesquisas em situaçóes mais naturais, tais como a escola, assim como o envolvimento do professor no ensino utilizando o PECS.

Essa pesquisa, como qualquer outra, apresentou limitaçóes: o tipo de revisão adotado (revisão sistematizada), embora consiga apresentar os resultados de maneira sistemática, não possui algumas características presentes nas revisóes sistemáticas, como a avaliação da qualidade das pesquisas como critério para inclusão e a presença de dois observadores. Além disso, é evidente que o número de bases de dados pesquisado, o período de tempo, os descritores 
utilizados, estão diretamente relacionados com o número de trabalhos encontrados. Dessa forma, pesquisas que ampliem os campos pesquisados também são benvindas, assim como revisóes sistemáticas, que permitam um olhar mais compreensivo dos resultados encontrados nessa pesquisa.

\section{Conclusão}

Essa revisão mostrou que o PECS, um método de CAA, em consonância com a literatura existente sobre o assunto, parece ser um promotor de comunicação gestual e vocal em indivíduos com TEA/TGD e/ou dificuldades de fala, apesar de indicar ser uma estratégia de ensino individual.Os ganhos obtidos parecem ser inicialmente as trocas de figuras, realizadas de maneira independente, seguidas de vocalizaçóes e/ou aproximaçóes de palavras com intenção comunicativa, chegando à fala funcional em alguns casos. Entretanto, dados sobre o que é necessário para ter sucesso nas fases, assim como aspectos do treino que devem existir para promover a comunicação via PECS ainda permanecem obscuros, o que justifica a continuidade de pesquisas sobre o PECS.

\section{REFERÊNCIAS}

ALMEIDA, M.A.; PIZZA, M.H.M.; LAMÔNICA, D.A.C. Adaptaçôes do sistema de comunicação por troca de figuras no contexto escolar. Pró-Fono Revista de Atualização Científica, Barueri, v.17 n.2, p.233-240, 2005.

ASSOCIAÇÃO AMERICANA DE PSIQUIATRIA. Manual Diagnóstico e Estatístico dos Distúrbios Mentais - DSM-IV-TR. 4. ed. Revisado, 2003.

BONDY, A.; FROST, L. The Picture Exchange Communication System. Behavior Modification, v.25, n.5, p.725-744, 2001.

CARR, D.;FELCE, J. Brief Report: Increase in Production of Spoken Words in Some Children with Autism after PECS Teaching to Phase II. Journal of Autism and Developmental Disorders, New York, v.37, n.4, p.780-787, 2007.

CATANIA, A.C. Aprendizagem: Comportamento, linguagem, cognição. Trad. Deisy das Graças de Souza (et. al.). 4. ed. Porto Alegre: Artes Médicas, 1999.

CHAABANE, D.B.B.; ALBER-MORGAN, S.R.;DEBAR, R.M. The Effects of parent-implemented PECS training on improvisation of mands by children with autism. Journal of Applied Behavior Analysis, v.42, n.3, p. 671-677, 2009.

COWAN R.J.; ALLEN, K.D. Using naturalistic procedures to enhance learning in individuals with autism: a focus on generalized teaching within the school setting. Psychology in the Schools,v.44, n.7, p.701-715, 2007.

FERREIRA, P.R.; TEIXEIRA, E.V.S.;BRITO, D.B.O. Relato de Caso: Descrição da evolução da comunicação alternativa na pragmática do adulto portador de autismo. Revista CEFAC: Atualização Científica em Fonoaudiologia e Educação, São Paulo, v.13, n.3, p.559-567, 2011. 
FIDALGO, A. P.; GODOI, J.P.; GIOIA, G.S. Análise de um procedimento de comunicação funcional alternativa (Picture Exchange Communication System). Revista Brasileira de Terapia Comportamental e Cognitiva, Belo Horizonte, v.10, n.1. p.51-66, 2008.

FLIPPIN, M.; RESKA, S.;WATSON, L.R. Effectiveness of the Picture Exchange Communication System (PECS) on Communication and Speech for Children With Autism Spectrum Disorders: A Meta-Analysis. American Journal of Speech-Language Pathology, Rockville, v.19, n.2, p.178-195, 2010.

FOSTER, S.L.; MASH, E.J. Assessing Social Validity in Clinical Treatment Research Issues and Procedures. Journal of Consulting and Clinical Psychology, v.67, n.3, p.308-319, 1999.

GANZ, J.B.; PARKER, R.; BENSON, J. Impact of the Picture Exchange Communication System: Effects on Communication and Collateral Effects on Maladaptive Behaviors. Augmentative and Alternative Communication, Baltimore, v.25, n.4, p.250-261, 2009.

GANZ, J.B.; SIMPSON, R.L. Effects on Communicative Requesting and Speech Development of the Picture Exchange Communication System in Children With Characteristics of Autism. Journal of Autism and Developmental Disorders, New York, v.34, n.4, p.395-409, 2004.

GANZ, J.B.; SIMPSON, R.L.; CORBIN-NEWSOME, J. The impact of the Picture Exchange Communication System on requesting and speech development in preschoolers with autism spectrum disorders and similar characteristics. Research in Autism Spectrum Disorders, v.2, n.1, p.157-169, 2008.

GRANT, M.J.; BOOTHT, A. A typology of reviews: an analysis of 14 review types and associated methodologies. Health Information and Libraries Journal, v. 26, n. 2, p.91-108, 2009.

LEITE, M.T.L. Aquisição e Generalização de Mandos em uma Criança com Autismo. 2005. 105 f. Dissertaçáo (Mestrado em Psicologia do Desenvolvimento). Departamento de Psicologia. Universidade Católica de Goiás, Goiânia. 2005.

MALHOTRA, S.; RAJENDER, G.; BHATIA, M.S.; SINGH, T.B. Effects of Picture Exchange Communication System on Communication and Behavioral Anomalies in Autism. Indian Journal of Psychological Medicine, v.32, n.2, p.141-143, 2010.

NUNES, D.R.P. Introdução. In: MANZINI, E.J. et al. Linguagem e comunicação alternativa. 1. ed. Londrina: ABPEE, 2009. p. 1-8.

NUNES, L.R.O.P. Favorecendo o desenvolvimento da comunicação em crianças e jovens com necessidades educacionais especiais. 1. ed. Rio de Janeiro: Dunya, 2003.

- Construção de sentenças com uso de sistema pictográfico de comunicação alternativa: contribuição para estudos sobre memória e desenvolvimento da sintaxe. In: MENDES, E.G.; ALMEIDA, M.A.; HAYASHI, M.C.P.I. (Org.). Temas em educação especial: conhecimentos para fundamentar a prática. 1. ed. Araraquara: Junqueira \& Marin, 2008. p.145-159.

PIZA, M.H.M. O uso dos métodos alternativos PECS-Adaptado e PCS para aumentar habilidades comunicativas em paralíticos cerebrais, não verbais. 2002.102 f. Dissertação (Mestrado em Educação Especial). Departamento de Psicologia, Universidade Federal de São Carlos, São Carlos. 2002.

PRESTON, D.; CARTER, M. A Review of the Efficacy of the Picture Exchange Communication. Journal of Autism and Developmental Disorders, New York, v.39, n.10, p.1471-1486, 2009.

WALTER, C.C.F. Os efeitos da adaptação do PECS associada ao curriculum funcional natural em pessoas com autismo infantil. 2000. 89f. Dissertação (Mestrado em Educação Especial). Departamento de Psicologia, Universidade Federal de São Carlos, São Carlos. 2000. 
WALTER, C.C.F. Avaliação de um programa de comunicação alternativa e ampliada aplicado por mães de adolescentes com autismo. 2007. 110f. Tese (Doutorado em Educação Especial). Departamento de Psicologia, Universidade Federal de São Carlos, São Carlos. 2007.

YODER, P.J.; LIEBERMAN, R.G. Brief Report: Randomized Test of the Efficacy of Picture Exchange Communication System on Highly Generalized Picture Exchanges in Children with ASD. Journal of Autism and Developmental Disorders, New York, v.40, n.5, p.629-632, 2010. 